Ulrich Braukämper (2009): Zum Widerstreit symbolistischer und kulturmaterialistischer Ansätze in der Deutung von Nahrungstabus. In: Elfriede Hermann, Karin Klenke und Michael Dickhardt (Hg.): Form, Macht, Differenz. Motive und Felder ethnologischen Forschens. Göttingen: Universitätsverlag Göttingen, 319-329. Doi: 10.17875/gup2020-1294

\title{
Zum Widerstreit symbolistischer und kulturmaterialistischer Ansätze in der Deutung von Nahrungstabus
}

\author{
Ulrich Braukämper
}

\section{Vorbemerkungen}

Die Bedeutung von Nahrung kann wohl kaum prägnanter zusammengefasst werden als in dem folgenden Satz von Carole Counihan: „Food is a many-splendored thing, central to biological and social life" (1999:6). Über ihre unmittelbare physiologische Bedeutung als "primary need" hinaus übt sie tief greifende Einflüsse auf Konzepte von Körperlichkeit und Ästhetik, Gender, gesellschaftlich-politische Statuspositionen und weltanschaulich-religiöse Orientierungen aus. Die „Ethnologie der Ernährung" (nutritional anthropology) ist bestrebt, den überall auf der Welt kulturspezifischen Umgang mit Nahrungsmitteln in einer sowohl analytischen als auch komparatistischen und verstehenden Perspektive zu untersuchen. In diesem Forschungsbereich hat Brigitta Hauser-Schäublin (1986) einen Beitrag geleistet, der ihr Leben „im Feld“ veranschaulicht ${ }^{1}$ und gleichzeitig auch das facettenreiche Mit- und Gegeneinander von Natur und Kultur anspricht, ein Themenbereich, den sie in späteren Arbeiten (z. B. 2001) immer wieder in den Mittelpunkt ihres wissenschaftlichen Interesses stellte.

In diesen Ausführungen sollen Wertvorstellungen und Handlungen, die mit Nahrungsmitteln assoziiert sind, und davon wiederum Meidungen bzw. Tabus, im Blick- 
feld stehen. Den Ethnografen ${ }^{2}$ eröffnen auf Feldforschungen die Verhaltensweisen bei Mahlzeiten, die „Tischsitten“, häufig die ersten Einblicke in die Interaktionsmuster und die Hierarchie einer Gesellschaft. Während Vorlieben für bestimmte Nahrungsmittel unschwer zu erkennen sind und mitunter plakativ bis hin zur Spitznamengebung herausgestellt werden, erschließen sich negative Einstellungen und Tabus im Bereich des Essens durchweg erst nach intensiverer Vertrautheit mit fremden Kulturen. Mehr noch als die den Regional- und Nationalküchen entgegengebrachten Präferenzen sind sie mit Maßstäben westlicher Rationalität oft schwer zu begreifen. ${ }^{3}$ Beispielsweise konnte ich Anfang der 1990er Jahre in dem gemeinsam von Shuwa-Arabern und Kanuri bewohnten Dorf Kamzamo in Nordost-Nigeria beobachten, dass Erstere die Blätter des Baobab-Baumes (Adansonia Digitata L.) verschmähten und Letztere für den Verzehr schätzten. Generell ist die Nahrung der wichtigste Bereich des Konsums und gleichzeitig ein Teil der Kultur, in dem das Individuum „soziale Nachrichten“ senden und empfangen kann (Appadurai 1986:31).

Da der Geschmackssinn bei der Gattung Homo sapiens als im Wesentlichen gleichartig ausgeprägt gilt, ist im Prinzip davon auszugehen, dass kulturelle Kriterien über Wohlgeschmack und Widerwillen im Hinblick auf Nahrungsmittel entscheiden. Allerdings dürfen naturgegebene Faktoren wie die genetisch bedingte Unverträglichkeit von Milchzucker (Laktose) bei einem beträchtlichen Teil der Weltbevölkerung nicht unberücksichtigt bleiben. Untersuchungen darüber liegen beispielsweise für Populationen im Gebiet der großen Seen Ostafrikas vor (Cook 1969). Somit müssen Forschungen in der Ernährungsethnologie neben den für sie zentralen kulturwissenschaftlichen auch naturwissenschaftliche Erkenntnisse berücksichtigen und gewichten.

Die Charakteristika im Hinblick auf Meidungen von Nahrungsmitteln sind vielfältig: Alter, Geschlecht, gesellschaftlicher Status, religiöse und ethnische Zugehörigkeit des Menschen, zeitlicher und zeremonieller Anlass der Meidung sowie Art der tabuisierten Nahrungsmittel. Sie können zudem auch in den verschiedensten Kombinationen miteinander verknüpft sein. Auffallend ist die Häufung von Speiseverboten in den als krisenhaft geltenden Stadien des Lebenszyklus, wie z. B. Pubertät und Schwangerschaft. Die weit verbreitete Einteilung in „kalte“ und „heiße“ Nahrungsmittel, die in bestimmten Situationen, körperlichen oder seelischen Zuständen entweder vorzuziehen oder zu meiden sind (siehe z.B. Eichinger Ferro-Luzzi 1980:98f., Ladermann 1981:475f.), ist zwar eine im Grundsatz symbolische Klassifikation, die jedoch in Einzelbereichen durchaus aus Erfahrungen resultieren kann, da manche Substanzen, wie etwa Fisch, in bestimmten körperlichen Zuständen (z. B. Schwangerschaft) toxisch wirken können.

Da der Akzent auf Meidungen von Nahrungsmitteln liegen soll, ist ein definitorisches Problem anzusprechen: nicht alle Meidungen sind Tabus. Eine Meidung, wie z. B. die von Pferdefleisch im heutigen Nordeuropa, kann über individuelle Abneigungen hinaus auch kollektiv ausgeprägt sein, ohne dass ein normativer Zwang vorliegen müsste. Im Unterschied dazu setzt das seit Ende des 18. Jahrhunderts aus Polynesien in die abendländische Kulturwissenschaft übertragene Konzept des Tabus die Über- 
zeugung voraus, dass die Meidungsgebote von übernatürlichen Mächten sanktioniert wurden und jede Missachtung für das Individuum und seine Gruppe gefährlich ist (Steiner 1956:21). In diesem Sinne muss ein nicht unbeträchtlicher Teil der Nahrungsverbote sowohl in Gesellschaften mit unkodifizierten Volksreligionen als auch in Gesellschaften mit Schrift besitzenden Weltreligionen als „echte“ Tabus gelten. Mit einem Tabu werden in der Regel nur solche Speisen belegt, die integrierter Bestandteil eines kulturellen Systems sind. Unbekannte Nahrungsmittel werden zumeist spontan, oft mit Abscheu und Ekel, abgelehnt, ohne dass kulturelle Regulative dafür aufgeboten werden müssten. Mitunter ist jedoch ein Corpus von Vorschriften kodifiziert worden, wie etwa im Islam, der explizit zwischen harām im Sinne eines religiös sanktionierten Tabus und haläl, den erlaubten Speisen und Praktiken, unterscheidet.

Für die Deutung der Nahrungsverbote kommt der Ethnologie insofern eine Schlüsselrolle zu, als ihre Materialgrundlagen eine offenkundig größere phänomenologische Vielfalt und Mannigfaltigkeit aufzeigen als die der so genannten Hochkulturen. Einen bedeutenden Impuls für die Diskussion der Tabus gab die „Entdeckung“ des Totemismus vor allem bei Ureinwohnern Nordamerikas und Australiens. Die weltweit verbreitete Vorstellung, dass menschliche Gruppen ihren Ursprung auf ein Tier, eine Pflanze oder ein Naturphänomen zurückführen, ist als Faktum unbestreitbar, auch wenn Claude Lévi-Strauss (1968) berechtigte Zweifel an der überkommenen Interpretation des Totemismus-Komplexes anmeldete. Ein zentrales Element wurde einerseits darin gesehen, dass die Totem-Spezies von den mit ihr assoziierten Menschen nicht getötet werden durfte; andererseits war mitunter jedoch gerade ihr Verzehr in Form einer ritualisierten „Kommunion“ erstrebenswert. ${ }^{4}$

Der Beginn einer verstärkten wissenschaftlichen Debatte um die Deutung von Nahrungstabus in der Ethnologie, Soziologie und Religionswissenschaft lässt sich in die 1960er Jahre datieren. Eine Besonderheit in dieser Kontroverse, die ihre Aktualität bislang nicht eingebüßt hat, lag darin, dass sie sich zwischen zwei Extrempositionen vollzog, der symbolistischen (oder auch idealistischen) und der (kultur)materialistischen. Exemplarisch am deutlichsten lässt sich diese Auseinandersetzung am Werk der führenden Protagonisten der beiden Richtungen nachvollziehen: Mary Douglas (1921-2007) für den Symbolismus und Marvin Harris (1927-2001) für den Kulturmaterialismus.

\section{Der symbolistische Forschungsansatz}

1966 veröffentlichte Mary Douglas in dem Buch „Purity and Danger“5 ihre zentrale These, dass Reinheitsrituale und Tabus weltweit in kulturellen Wertvorstellungen gründen, die dazu dienen, das gesellschaftliche Leben in seiner Gesamtheit sinnvoll zu organisieren. Sie wurde zur namhaftesten Vertreterin einer Forschungsrichtung, die der Ausdeutung von Symbolen eine entscheidende Rolle für das Verständnis kultureller 
Tatbestände im weitesten Sinne zuweist und von idealistisch zu nennenden Grundsätzen ausgeht. ${ }^{6}$

Im Abendland ist das Verbot bestimmter Nahrungsmittel weniger aus dem eigenen Kulturbereich als durch den Kontakt mit orientalischen Weltreligionen, Judentum, christlich-orthodoxen Konfessionen und Islam ein seit langem vertrauter Kenntnisbereich. Bezeichnenderweise war es die Rezeption biblischer Texte, vor allem von Levitikus und Deuteronomium, des 3. und 5. Buches Mose, die Douglas' Interesse an der Thematik der Reinheitsgebote und damit auch den Nahrungstabus weckte. Sie argumentiert, dass alle bisher vorgebrachten Ausdeutungen der mosaischen Gesetze sich in zwei Gruppen aufteilen lassen: „entweder werden die Vorschriften als bedeutungslos und willkürlich hingestellt, weil es ihnen nicht darum geht, zu belehren, sondern zu disziplinieren, oder sie werden als Allegorien für Tugenden und Laster" (Douglas 1988:62) dargestellt. Sie führt weiter aus, dass alle Versuche, die Verbote des Alten Testamentes einzeln und losgelöst voneinander zu betrachten, notwendigerweise scheitern müssen und gelangt zu dem Schluss, dass es zwischen Heiligkeit und Gräuel so etwas wie eine Unvereinbarkeit gäbe, die den verschiedenen Einzelvorschriften einen übergreifenden Sinn verleihe (Douglas 1988:69).

Dieser Sinn sei aus dem Ordnungsprinzip der Genesis, nämlich der Einteilung in die drei Elemente Wasser, Luft und Erde, abzuleiten. Die Bibel wies jedem Element die ihm angemessene Art von Tieren zu: unter dem Firmament fliegen Tiere mit zwei Flügeln und zwei Füßen, im Wasser schwimmen Fische mit Flossen und Schuppen, auf der Erde laufen und hüpfen vierfüßige Tiere. Jede Spezies, der die für ihr Element als adäquat erkannte Fortbewegungsart fehlt, verstößt gegen das als sakrosankt empfundene Ordnungsprinzip und jeglicher Kontakt mit ihnen macht den Menschen „unrein“. Darunter fallen im Wasser lebende Säugetiere, Vögel, die nur laufen, und Kriechtiere. In diesen Bereichen erscheint die Begründung logisch stringent. Schwierig ist jedoch die Tatsache zu deuten, warum von den Paarhufern nur die Wiederkäuer als rein gelten. Die Israeliten waren Agropastoralisten, die ihren Lebensunterhalt zum Großteil aus den Produkten ihrer Herdentiere Schaf, Ziege und Rind bestritten. Was das Schwein als einen nicht wiederkäuenden Paarhufer angeht, so spielte es in ihrer Ökonomie keine Rolle und konnte deshalb relativ leicht ausgesondert werden. Hier nimmt Douglas offenkundig Zuflucht bei sonst von ihr verschmähten utilitaristischen Argumenten (vgl. unten).

Die Tatsache, dass das Fleisch von Wildtieren gemäß den Vorschriften der mosaischen Gesetze kaum zu nutzen war, versucht Douglas (1988:74f.) aus einer ethnologischen Analogie zu erklären. Die Jagd werde z.B. von ostafrikanischen Hirtenvölkern kaum praktiziert. Für das Kamel wird die Aussonderung aus der Kategorie der „reinen“ und damit essbaren Tiere, nur weil es kein Paarhufer ist, vollends problematisch. Warum konnte für dieses in ariden Gebieten so überaus nützliche Tier die starre Taxonomie nicht ebenso durchbrochen werden, wie es - etwa nach der Argumentation von Harris (1988:182f.) - im Falle der Heuschrecke geschah? Für die Muslime, die sich stark an die 
Tradition der alttestamentarischen Reinheitsgebote anlehnten, wurde das Verbot von Kamelfleisch und -milch explizit aufgehoben. Andernfalls hätte die Islamisierung für die arabischen Wüstennomaden das Ende ihrer Existenz bedeutet.

Auf ihrer Feldforschung bei den Lele im Kongo hatten Mary Douglas (1957:4658) die mit Tieren verbundenen Symbolvorstellungen offenbar so fasziniert, dass sie fortan ein als allgemeingültig zu verstehendes kognitives Modell aufzubauen bestrebt war. Es war dann ein folgerichtiger Schritt, in den für die universale Kulturgeschichte zentralen Schriftwerken wie der Bibel nach beweiskräftigen Analogien zu suchen.

Die Lele unterscheiden in ihrer Taxonomie Tagtiere und Nachttiere sowie Tiere, die oben leben, Vögel und Affen, und Tiere, die unten, d.h. auf dem Land und im Wasser leben. Tierarten, die sich nicht eindeutig zuordnen lassen, z. B. Flughörnchen oder Laufvögel, gelten als anomale Lebewesen und sind deshalb für erwachsene Menschen ungenießbar. Tiere, die in enger Gemeinschaft mit den Menschen leben, Hunde, Ziegen, aber auch Ratten und Geckos, werden ebenfalls nicht gegessen. Hier wird auch ein Gegensatz manifest, der in vielen Kulturen als „wild“ und „domestiziert" konzeptionalisiert wird. ${ }^{7}$ Die Welt der Menschen und die der Tiere und Naturphänomene stehen also in einem ganz bestimmten Bezug zueinander. Unter den Tieren der Wildnis, die im Prinzip als Nahrungsquelle dienen, haben jedoch jene Arten mit „anomalem“ Verhalten eine besondere Stellung, weil sie mit Geistwesen assoziiert werden und deshalb vom Verzehr ausgeschlossen sind. Douglas (1988:60-62, passim) sieht in diesen Meidungen die Reflexion eines logischen, von kognitiven Dissonanzen freien Weltbildes: Menschen und gesellschaftliche Zustände, die eine Gefahr für die soziale Ordnung bedeuten, finden eine symbolische Entsprechung in den zu meidenden Tierarten. Diese Prämisse hält sie sowohl für alle Kulturstufen als auch - durch die Bibel - für eine große Zeittiefe eindeutig nachweisbar. Die Richtigkeit der ethnologischen Bestandsaufnahme und Deutung der Lele-Kultur durch Mary Douglas voraussetzend, drängen sich jedoch massive Zweifel auf, ob die durch Untersuchungen bei einer Ethnie von Regenwaldbauern gewonnenen Erkenntnisse den Anspruch eines universal einsetzbaren Paradigmas erheben können.

\section{Der kulturmaterialistische Forschungsansatz}

Als ein zeitgenössischer Antipode zur symbolistischen Forschungsrichtung entstand der von kulturökologisch-szientistischen und marxistischen Ideen angeregte Kulturmaterialismus. Sein Begründer Marvin Harris hatte 1966 mit Thesen über die „heilige

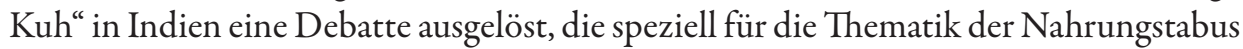
bedeutsam wurde. Er wendet sich gegen die weltweit vorherrschende Meinung, welche die hinduistischen Fleischtabus als einen "Triumph der Moral über den Appetit“ herausgestellt hat. Nach seiner Überzeugung besteht das Tötungs- und Verzehrverbot des Rindes für die Hindus nicht deshalb, weil das Tier eine Art Apotheose erfahren 
hat, sondern aus der Erkenntnis, Rinder könnten der indischen Agrargesellschaft am besten dadurch dienen, dass sie als Arbeitstiere zum Pflügen und Lastentransport sowie als Lieferanten von Milch und Dung in einer möglichst großen Zahl zur Verfügung stehen. Jede Schlachtung würde eine Gefährdung des Bestandes bedeuten und somit einer zweckrationalen Wirtschaftslogik widersprechen. Folgerichtig sind die reproduktiven Kräfte gegenüber den produktiven auf der ganzen Linie als vorrangig zu werten.

Ein weiteres für Harris' utilitaristische Erklärungsweise besonders typisches Beispiel ist die Ausnahme der Heuschrecke vom alttestamentlichen Gebot des Verzehrs von Insekten. Die Heuschrecke, so argumentiert er (1977:134), ist groß und fleischig und kommt in riesigen Schwärmen vor, kann als Nahrungsressource leicht eingesammelt werden und stellt außerdem eine Bedrohung für die Nutzflächen dar. Die Ausnahme von der Tabuisierung ist somit ein Gebot wirtschaftlicher Logik. Der Kannibalismus wurde nach Harris (1977, 1988:Kap.10) nicht aufgrund von Moralvorstellungen, sondern deshalb verfemt, weil die Erkenntnis siegte, dass der Mensch als Produktionsmittel besser nutzbar war als als Fleischlieferant. Wo dennoch Anthropophagie praktiziert wurde, wie bei den Azteken, soll dies wegen eines chronischen Eiweißmangels lebensnotwendig gewesen sein. Marshall Sahlins (1978:48f.) hat jedoch gerade an diesem Beispiel eklatante Fehlberechnungen von Harris aufgedeckt und überzeugend nachgewiesen, dass die Azteken auch ohne Menschenfleisch über eine gute Proteinversorgung verfügten.

Gewichtige Argumente zur Stützung ihres Theoriengebäudes glaubten die Kulturmaterialisten im Jagdverhalten indigener Gruppen zu finden. Bei zahlreichen Gruppen des amazonischen Regenwaldes wie etwa den Matsigenka, Desana, Kayapó und Shuar ist die Vorstellung verbreitet, dass Tiere, die selber jagen und töten, Feliden, Schlangen, Raubvögel, Kaimane, nicht essbar seien (McDonald 1977:735-738, Ross 1978:5f., Baer 1986:26-30.). Bei dem verbleibenden Jagdwild unterliegen häufig Hirsche, seltener Tapire, einem Tötungsverbot, da sie nach gängiger Auffassung als Träger menschlicher Seelen angesehen werden. Dieser religiös begründeten Deutung setzen Vertreter der kulturmaterialistischen Schule, vor allem McDonald (1977) und Ross (1978), die Überzeugung entgegen, dass solche Tabus eine zielgerichtete Maßnahme zum Erhalt des ökologischen Gleichgewichtes sind. Große Tierspezies seien einerseits im Regenwald weniger zahlreich als etwa in der Savanne und wiesen andererseits eine so geringe Reproduktionsrate auf, dass eine Bejagung ihren Bestand gefährden würde. Jedoch weisen die Autoren selbst darauf hin, dass die Waldlandbewohner die jägerische Komponente in ihrem kulturellen Selbstverständnis zwar hervorheben, ihre Ernährung jedoch zu zwei Dritteln aus Produkten von Sammeltätigkeit, Fischfang und Gartenbau bestreiten. Die Wertschätzung der Jagd übertrifft unzweifelhaft also ihre reale Bedeutung für den Nahrungserwerb (siehe z. B. Ross 1978:4f., Baer 1986:30), so dass die Gefahr einer Übernutzung des Wildbestandes eher begrenzt ist.

Dem Ziel einer Harmonie von Mensch und Umwelt dient nach kulturmaterialistischer Auffassung auch das in jägerischen und bäuerlichen Ethnien weit verbreitete Pro- 
tein-Tabu für Schwangere. Es soll angeblich durch die daraus resultierende Erhöhung der Säuglingssterblichkeit eine demografische Auslese bewirken, die den vorhandenen Ressourcen angemessen ist. Wie ist das jedoch vereinbar mit dem sehr viel häufiger dokumentierten Bestreben, die eigene Gruppe durch eine möglichst hohe Geburtenrate und gegebenenfalls durch Adoptionen auszuweiten? ${ }^{8}$

Wie Mary Douglas hat auch Marvin Harris umfassend Vergleichsmaterialien aus den antiken Kulturen und den Weltreligionen zur Stützung seines Theoriengebäudes herangezogen. Ein exponiertes Beispiel ist das Schweinefleisch-Tabu, das Judentum, Islam, einige christlich-orthodoxe Konfessionen sowie davon beeinflusste Stammesreligionen, z. B. in Süd-Äthiopien, gemeinsam haben. Nach Douglas (1988:75) wurde das Schwein für die Israeliten deshalb ein unreines Tier, weil sie es als eine „taxonomische Anomalie“ nicht bei den „reinen“ Tieren unterbringen konnten. Demgegenüber versucht Harris (1988:75f.) darzulegen, dass der einstmals blühenden Schweinezucht im palästinensisch-syrischen Raum durch die Vernichtung der Wälder seit etwa 5000 v. Chr. die ökologische Grundlage entzogen wurde. Das Tier wurde dann als Bedrohung für die Landwirtschaft und als Nahrungskonkurrent des Menschen zwangsläufig verfemt und ausgesondert. Die Meidung von Pferdefleisch in Teilen Europas ist nach Harris (1988:96-99) nicht dadurch begründet, dass es während der Christianisierung des frühen Mittelalters als ein Kernsymbol „heidnischer" Praktiken geächtet werden sollte, sondern weil Pferde für den Aufbau einer Reiterei zur Verteidigung des Abendlandes gegen die Muslime überlebenswichtig wurden.

\section{Kritischer Vergleich der symbolistischen und kulturmaterialistischen Positionen}

Die symbolistische und die kulturmaterialistische Schule vertreten in einer sehr apodiktisch erscheinenden Weise Standpunkte, die summarisch in den folgenden Hauptpunkten zusammengefasst werden können. Für Mary Douglas stehen als Prämissen und Deutungsmuster im Blickpunkt: Priorität des menschlichen Geistes vor materialistischen Tatbeständen, Streben nach einer holistischen Kulturbetrachtung, Teleologie eines rituellen „Reinheitsverständnisses“, zentrale Bedeutung von Symbolen, „fundamentalistisch" erscheinende Exegese religiöser Schriften. Die Positionen von Marvin Harris stehen dazu in diametralem Gegensatz: Priorität materieller Umweltgegebenheiten, utilitaristische Teleologie, partikularistische ökologische Adaption, Vorrang reproduktiver gegenüber produktiven Faktoren, pragmatisches Tatsachenverständnis, positivistisch-szientistische Erkenntnistheorie.

Wie überall in kultur- und sozialwissenschaftlichen Themenbereichen und Diskursen monokausale Erklärungen zweifelhaft sind, kann auch die Deutung von Nahrungstabus entweder im Sinne der symbolistischen oder der kulturmaterialistischen Richtung nicht überzeugend sein. Für eine differenzierte Erklärung erscheint deshalb eine eklekti- 
sche Vorgehensweise unabdingbar - wobei ich mir bewusst bin, dass Begrifflichkeit und Konzept von „eklektisch“ im Grundsatz nicht als positiv konnotiert gelten. Es spricht zunächst einmal nichts dagegen, dass Speisetabus sowohl im ökobiologischen Bereich, als auch im religiösen Denken und Handeln einer Gruppe verwurzelt sein können, und je mehr Funktionen sie entsprechen, desto größer wird offenkundig ihre Bedeutung für die Gesamtkultur. Wie ich selbst in Äthiopien beobachten konnte (Braukämper 1984:441f.), liegt eine zentrale Rolle der Nahrungstabus, zu denen auch Fastengebote zählen, darin, die Identität von Gruppen auf verschiedenen Ebenen, sozio-politisch, religiös, ethnisch, abzugrenzen. Je ausgeprägter sie sind, desto höher ist in der Regel die gesellschaftliche Position der ihnen unterworfenen Individuen, eine Tatsache, die etwa auch im indischen Kastensystem bestätigt wird. Andererseits ist das auffallend häufig auf schwächere Bestandteile der Gesellschaft wie Kinder, Frauen, Hörige ausgerichtete Verbot hochwertiger Nahrungsmittel als ein die Gruppenhierarchie sanktionierendes Machtinstrument zu deuten.

Die vornehmlich in Südamerika exemplifizierte These der Kulturmaterialisten, dass gute Fleischlieferanten aus umweltbewusster Ratio geschont werden, kann insofern nicht überzeugen, als bei den Pygmäen des zentralafrikanischen Regenwaldes gerade die großen und keineswegs häufig vorkommenden Tierarten wie Elefanten, Rotbüffel, Großantilopen und Gorillas als Jagdbeute durchaus begehrt sind. Dem Argument, die Tötungstabus für Wildtiere resultierten aus der Einsicht, dass nur so der Erhalt ihrer Arten und damit das Gleichgewicht des Ökosystems gewahrt werden könne, wie auch Roy Rappaport (1967:26) mit seinem neo-funktionalistischen Ansatz betont, widerspricht die Tatsache, dass Menschen einer jägerisch-bäuerlichen Kulturstufe durchaus an der Reduzierung oder gar Vernichtung von Tierspezies beteiligt sein konnten. Ein Beispiel dafür ist die Ausrottung des neuseeländischen Riesenvogels Moa durch die Maori im 18. Jahrhundert.?

Beim Jagdverhalten erscheint mir die symbolistische Interpretation, dass Tiere als vermeintliche Träger von Geistwesen geschont werden, plausibler als die kulturmaterialistische Erklärung. Allerdings ist der Analogieschluss von Mary Douglas, dass der weitgehende Verzicht ostafrikanischer Hirtenvölker auf Jagdwild die biblischen Tabuvorschriften in diesem Bereich gewissermaßen bestätigt, nur von begrenztem Aussagewert. Was die Stigmatisierung von Kannibalismus und den Verzehr von Pferdefleisch angeht, so ist auch hier nach dem in der wissenschaftlichen Literatur ganz überwiegend geäußerten Argumentationsstand die religiöse Begründung wesentlich überzeugender als die utilitaristische. Hingegen entbehrt im Falle des Schweinefleisch-Tabus das historisch begründete wirtschaftlich-rationale Deutungsmuster von Harris nicht der Überzeugungskraft. Es ist jedoch nicht abwegig, dass gleichzeitig - oder nachfolgend - auch ein Reinheitsdenken zu der Meidung beigetragen haben kann. Das Wissen über Trichinella spiralis und die Übertragung des Parasiten vom Schwein auf den Menschen konnten die Schöpfer der mosaischen Gesetze zwar nicht besitzen, da die Zusammenhänge erst im 19. Jahrhundert bekannt wurden. Man kann jedoch wohl eine im Laufe von 
Generationen erworbene Erfahrung als gegeben annehmen, dass nach dem Verzehr von Schweinefleisch eine bestimmte Krankheit vermehrt auftrat. Für die Tabuisierung wäre in der Tat wohl ein Zusammenwirken ökologisch-ökonomischer Veränderungen und in kulturellen Wertvorstellungen gründender Reinheitsvorstellungen anzunehmen.

Ein idealistischer Zugang impliziert keinesfalls die Abkehr von einer empirischen Forschungsstrategie. Was letztlich die gelebte Wirklichkeit bestimmt, sind die kognitiven Konzepte, mit denen menschliche Gruppen ihrer eigenen Umwelt entgegenstehen und sie zu gestalten versuchen. Ob diese von außen stehenden Beobachtern mit Kategorisierungen wie rational oder irrational, symbolistisch oder materialistisch bedacht werden, ist für die entsprechenden Menschen selbst belanglos. Wenn etwa die Kulturmaterialisten den Indigenen eine zielbewusst verfolgte Strategie zur Aufrechterhaltung des ökologischen Gleichgewichtes zuerkennen, so ist das eine zwar ehrenwerte Argumentation, die oft jedoch den Tatsachen widerspricht und den menschlichen Geist in eine utilitaristische Zwangsjacke einengt. Es gibt nach meiner Überzeugung umfassende Handlungsspielräume, die Menschen verschiedenster Kulturausstattung befähigt, auf äußere und oft unvorhersehbare Einflüsse elastisch zu reagieren.

\section{Anmerkungen}

1 Nach den mündlichen Überlieferungen des Göttinger Instituts für Ethnologie pflegte sie beispielsweise zu betonen, dass die Grenzen ihrer eigenen kulinarischen Belastbarkeit mit der Fettdrüse eines Krokodils erreicht seien.

2 Der Einfachheit halber soll die maskuline Form hier durchgehend für beide Geschlechter verwendet werden.

3 Was die Verhaltensweisen im Hinblick auf Nahrung angeht, so waren für mich selbst die Feldforschungen bei Ethnien Süd-Äthiopiens in den 1970er Jahren eine besonders prägende Erfahrung (vgl. Braukämper 1984).

4 Die Verbindung, in die Sigmund Freud (1956[1913]) die Konzepte von Totem und Tabu in einer seinerzeit einflussreichen Schrift brachte, wird von der modernen Forschung als nicht mehr haltbar abgelehnt.

5 Im Folgenden beziehe ich mich auf die deutsche Übersetzung „Reinheit und Gefährdung“ von 1988.

6 Eine ähnlich exponierte Rolle, die der Nahrung auch im Strukturalismus von Lévi-Strauss (z. B. 1971) zukommt, soll hier außerhalb der Betrachtung bleiben.

7 Mit dieser Frage hat Brigitta Hauser-Schäublin (2001:15,17) sich u. a. im Kontext von Natur und Kultur als Machtbegriffe auseinandergesetzt.

8 Bei allen afrikanischen Ethnien, bei denen ich selbst geforscht habe, war das Ideal einer möglichst großen Zahl von Nachkommen ausgeprägt. Für das Bestreben einer Ausweitung durch Adoptionen sind vor allem Gruppen mit segmentären Lineage-Systemen, wie die Nuer im Sudan, bekannt geworden.

9 Ein Raubbau („overkill“) an den Ressourcen jagdbarer Tiere geht offenbar schon bis in die Zeiten prähistorischer Wildbeuter zurück (vgl. Cohen 1977). 


\section{Literatur}

Appadurai, Arjun (1986) Introduction: Commodities and the Politics of Value. In: Arjun Appadurai (Hg.) The Social Life of Things. Commodities in Cultural Perspective, S.3-63. Cambridge: University Press.

Baer, Gerhard (1986) Jagdverhalten und Nahrungstabus bei den Matsigenka-Indianern. In: Brigitta Hauser-Schäublin (Hg.): Rund ums Essen, S. 23-30. Basel: Brinkhäuser (Mensch, Kultur, Umwelt 1).

Braukämper, Ulrich (1984) On Food Avoidances in Southern Ethiopia: Religious Manifestation and Socio-Religious Relevance. In: Sven Rubensen (Hg.): Proceedings of the Seventh International Conference of Ethiopian Studies, S. 429-445. Lund: University of Lund.

Cohen, Mark N. (1977) The Food Crisis in Prehistory. Overpopulation and the Origin of Agriculture. New Haven: Yale University Press.

Cook, G.C. (1969) Lactase Deficiency: A Probable Ethnological Marker in East Africa. Man 4(2):265-267.

Counihan, Carole (1999) The Anthropology of Food and Body: Gender, Meaning and Power. New York: Routledge.

Douglas, Mary (1957) Animals in Lele Religious Symbolism. Africa 27(1):46-58.

Douglas, Mary (1988) Reinheit und Gefährdung. Eine Studie zu Vorstellungen von Verunreinigung und Tabu. Frankfurt a.M.: Suhrkamp.

Eichinger Ferro-Luzzi, Gabrielle (1980) Food Avoidances at Puberty and Menstruation in Tamilnad. In: John R. K. Robson (Hg.): Food, Ecology and Culture, S. 93-100. New York et.al.: Gordon and Breach.

Freud, Sigmund (1956) Totem und Tabu. Einige Übereinstimmungen im Seelenleben der Wilden und der Neurotiker. Frankfurt a.M.: Fischer.

Harris, Marvin (1966) The Cultural Ecology of India's Sacred Cattle. Current Anthropology 7(1):51-66.

Harris, Marvin (1977) Cannibals and Kings. The Origins of Cultures. New York: Random House.

Harris, Marvin (1988) Woblgeschmack und Widerwillen. Die Rätsel der Nahrungstabus. Stuttgart: Klett-Cotta.

Hauser-Schäublin, Brigitta (1986) Guten Appetit! - Guten Appetit? In: Brigitta Hauser-Schäublin (Hg.): Rund ums Essen, S. 5-14. Basel: Brinkhäuser (Mensch, Kultur, Umwelt 1). 
Hauser-Schäublin, Brigitta (2001) Von der Natur in der Kultur und der Kultur in der Natur. Eine kritische Reflexion dieses Begriffspaares. In: Rolf Wilhelm Brednich, Annette Schneider und Ute Werner (Hg.): Natur - Kultur. Volkskundliche Perspektiven auf Mensch und Umwelt, S. 11-20. Münster: Waxmann.

Laderman, Carol (1981) Symbolic and Empirical Reality: A New Approach to the Analysis of Food Avoidances. American Ethnologist 8(3):468-493.

Lévi-Strauss, Claude (1968) Das Ende des Totemismus. Frankfurt a. M.: Suhrkamp.

Lévi-Strauss, Claude (1971) Das Rohe und das Gekochte. Frankfurt a. M.: Suhrkamp (Mythologica 1).

McDonald, David R. (1977) Food Taboos: A Primitive Environmental Protection Agency (South America). Anthropos 72:734-748.

Rappaport, Roy (1967) Ritual Regulation of Environmental Relations among a New Guinea People. Ethnology 6:17-30.

Ross, Eric B. (1978) Food Taboos, Diet and Hunting Strategy: The Adaptation to Animals in Amazon Cultural Ecology. Current Anthropology 19(1):1-36.

Sahlins, Marshall (1978) Culture as Protein and Profit. The New York Review of Books 25(18):45-53.

Steiner, Franz (1956) Taboo. London: Cohen and West. 